\title{
Assessing the Value of Sarilumab Monotherapy for Adults with Moderately to Severely Active Rheumatoid Arthritis: A Cost-Effectiveness Analysis
}

\author{
Melanie D. Whittington, PhD; R. Brett McQueen, PhD; Daniel A. Ollendorf, PhD; \\ Richard H. Chapman, PhD; Varun M. Kumar, MBBS, MPH, MSc; Patricia G. Synnott, MS, MALD; \\ Foluso Agboola, MBBS, MPH; and Jonathan D. Campbell, PhD
}

\begin{abstract}
BACKGROUND: Rheumatoid arthritis is associated with a societal burden greater than $\$ 39$ billion annually. Novel treatments, known as targeted immune modulators (TIMs), are expensive but effective, producing improvements in response rates compared with conventional diseasemodifying antirheumatic drugs (cDMARDs). Sarilumab, a TIM approved in 2017, shows superior improvements compared with cDMARDs and produced significantly greater likelihood of achieving response and improvement in the Health Assessment Questionnaire Disability Index than adalimumab monotherapy. Although sarilumab monotherapy has shown improvements over cDMARDs and the TIM market leader adalimumab, treatment with sarilumab is costly, with an annual wholesale acquisition cost of $\$ 39,000$.
\end{abstract}

OBJECTIVE: To estimate the lifetime cost-effectiveness of starting treatment with sarilumab monotherapy for adult patients with moderately to severely active rheumatoid arthritis who have had an inadequate response to cDMARDs.

METHODS: A sequential treatment cohort model followed a hypothetical cohort from initiation of sarilumab monotherapy until death. The model allowed patients to switch therapies up to 3 times due to effectiveness or adverse events. The first switch was to a TIM within the same treatment category; the second switch was to a TIM within a different treatment category; and the third switch was to a cDMARD. Sarilumab monotherapy was compared with a cDMARD (methotrexate) and the TIM market leader (adalimumab monotherapy). Key risk and benefit evidence came from clinical studies and network meta-analyses of data on radiographic progression and response. We used a lifetime time horizon and the U.S. health sector payer perspective assuming therapy net pricing. We also incorporated loss of productivity to reflect a restricted societal perspective.

RESULTS: Over a lifetime time horizon, a treatment pathway starting with sarilumab resulted in 17.16 life-years and 13.66 quality-adjusted life-years (QALYs). Treatment pathways starting with the cDMARD resulted in 16.54 life-years and 11.77 QALYs; treatment pathways starting with adalimumab resulted in 17.05 life-years and 13.35 QALYs. Total costs for sarilumab ( $\$ 492,000$ for payer perspective, $\$ 634,000$ for societal perspective) were less than total costs for adalimumab $(\$ 536,000$ for payer perspective, $\$ 689,000$ for societal perspective) but higher than total costs for the cDMARD ( $\$ 63,000$ for payer perspective, $\$ 272,000$ for societal perspective). When compared with cDMARD therapy, sarilumab resulted in a cost-effectiveness estimate of $\$ 227,000$ per QALY gained from the payer perspective and $\$ 191,000$ per QALYs gained from the societal perspective. When compared with adalimumab, sarilumab was dominant from both perspectives.

CONCLUSIONS: Sarilumab resulted in better health outcomes than conventional therapy alone. However, its additional cost with assumed class-level net prices led to cost-effectiveness estimates above commonly cited thresholds. When compared with the market leader, sarilumab achieved favorable value. This evaluation informs stakeholders of the value of sarilumab and its alternatives to promote high value practices in health care.

J Manag Care Spec Pharm. 2019;25(1):80-87

Copyright $\odot 2019$, Academy of Managed Care Pharmacy. All rights reserved.

\section{What is already known about this subject}

Sarilumab monotherapy is superior to cDMARDs and adalimumab in achieving response, with additional improvements in the Health Assessment Questionnaire Disability Index.

With an annual wholesale acquisition cost of $\$ 39,000$, treatment using sarilumab is costly.

\section{What this study adds}

When compared with conventional therapy assuming class-level net pricing, cost-effectiveness estimates for sarilumab monotherapy were greater than commonly cited thresholds.

When compared with adalimumab monotherapy, sarilumab was dominant (i.e., less costly and more effective).

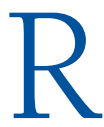
heumatoid arthritis (RA), which affects between 1.3 and 1.8 million Americans, is the most common chronic inflammatory arthritis in adults. ${ }^{1,2}$ This disease is typically characterized by stiffness and symmetrical joint swelling of the feet, hands, and knees, although any joint can be affected. ${ }^{3} \mathrm{RA}$ is associated with a significant economic burden resulting from excess health care utilization, quality of life deterioration, and premature mortality. The annual U.S. health care burden for RA is estimated to be $\$ 8.4$ billion, with an additional $\$ 10.9$ billion spent on RA-related consequences. ${ }^{4}$ When quality of life deterioration ( $\$ 10.3$ billion) and premature mortality ( $\$ 9.6$ billion) are included, RA's societal burden is greater than $\$ 39$ billion annually. ${ }^{4}$

Medications are available to manage RA and are distinguished by whether they treat the symptoms or whether they target the mechanisms of tissue damage. ${ }^{5}$ The latter 


\section{Assessing the Value of Sarilumab Monotherapy for Adults with Moderately to Severely Active Rheumatoid Arthritis: A Cost-Effectiveness Analysis}

variants are collectively referred to as disease-modifying antirheumatic drugs (DMARDs). ${ }^{5}$ Conventional DMARDs (cDMARDs) are older systemic agents that have broad immunomodulatory effects. Methotrexate, a widely used cDMARD, has been considered the "anchor drug" because of its effectiveness and tolerability; however, only about $50 \%$ of patients treated with methotrexate alone experience sufficient reduction in disease activity or symptoms, ${ }^{3}$ often characterized as a Disease Activity Score (DAS28) of 3.2 or less, with reductions in DAS28 of more than 1.2. ${ }^{6}$ This lack of response has led to the development of newer biologic and nonbiologic agents that target mediators of inflammation in RA. These novel agents are collectively referred to as targeted immune modulators (TIMs). Compared with cDMARDs, TIMs produce statistically and clinically superior improvements in American College of Rheumatology (ACR) defined response rates (i.e., 20\%, 50\%, and $70 \%$ improvement), disease activity, and radiographic progression. ${ }^{7}$ However, TIMs are expensive, with an average annual wholesale acquisition cost (WAC) of approximately $\$ 45,000 .{ }^{8}$ Evidence has shown that their prices and the proportion of the costs paid by patients have increased substantially in recent years. ${ }^{9}$

Sarilumab, a new TIM that received U.S. Food and Drug Administration approval in May 2017, is an interleukin-6 receptor antagonist indicated for adults with moderately to severely active RA with an inadequate response or intolerance to 1 or more cDMARDs. ${ }^{10}$ In addition to the superior improvements observed in comparison with cDMARDs, ${ }^{7,11}$ sarilumab monotherapy produced significantly greater likelihoods of achieving ACR response, clinical remission, and improvement in the Health Assessment Questionnaire Disability Index (HAQ) than adalimumab monotherapy, the TIM market leader.,12 Although sarilumab monotherapy has been shown to be more effective than CDMARDs and adalimumab monotherapy, treatment with sarilumab monotherapy is costly, with an annual WAC of $\$ 39,000 .{ }^{8}$ This is more than 30 times the annual WAC of largely generic cDMARDs, which is $\$ 1,155$ yet is priced less than the TIM market leader, with an annual WAC of approximately $\$ 58,000 .^{8}$

The objective of this analysis was to estimate the lifetime cost-effectiveness of sarilumab monotherapy for adult patients with moderately to severely active RA and an inadequate response to cDMARDs. This evaluation provides an assessment of the value of this recently approved TIM.

\section{Methods}

\section{Design}

To estimate the incremental cost per quality-adjusted lifeyear (QALY) gained with sarilumab monotherapy treatment, a sequential treatment cohort model followed a hypothetical cohort from initiation of sarilumab until death. ${ }^{7}$ Due to the chronic nature of RA, a lifetime time horizon was used. Key risk and benefit evidence were taken from clinical studies and network meta-analyses of data on radiographic progression and ACR response. ${ }^{\top}$ The deterministic results are reported from the U.S. health sector payer perspective and societal perspective. The health sector payer perspective included direct health care-related costs and effects borne by third-party payers or integrated health systems. The restricted societal perspective included productivity costs to the patient. All costs (except drug costs) were adjusted to 2016 U.S. dollars. All future costs and outcomes were discounted at 3\% per year.

\section{Population}

The population for this analysis included adults with moderately to severely active RA with an inadequate response or intolerance to cDMARDs. Moderate to severe RA was as defined in the randomized controlled trials for each TIM. The population in the clinical evidence was mostly TIM-naive, but TIM-experienced patients were not excluded from our analysis if they were included in the clinical evidence. The model simulated a hypothetical homogenous cohort of patients with baseline characteristics similar to typical patients in U.S. RA registries. ${ }^{13}$ The modeled cohort was mostly women (79\%), with an average age of 55 years. A full description of model cohort characteristics can be found in Appendix A (available from the authors at http://www.ucdenver.edu/academics/colleges/ pharmacy/Departments/ClinicalPharmacy/DOCPFaculty/A-G/ Pages/CampbellJonathanDPhD.aspx). Of these model cohort characteristics, age and gender were used in calculating the risk of mortality. The mean weight was used to calculate average dosing for TIMs administered intravenously, and the baseline HAQ score and Total Sharp Score (TSS) served as the starting point for the model-simulated HAQ score.

\section{Interventions}

The primary intervention of interest was sarilumab $200 \mathrm{mg}$ by subcutaneous injection every 2 weeks as monotherapy (i.e., not in combination with a cDMARD). In the clinical setting, it is common for patients to cycle through multiple therapies before finding a treatment option to which they best respond and tolerate. Therefore, other TIMs and cDMARDs were included in the model as sequential treatments after sarilumab. If TIMs had evidence for combination therapy (in combination with a cDMARD) and monotherapy (not in combination with a cDMARD), the approach with the higher treatment response was modeled.

\section{Comparators}

The sarilumab treatment pathway was compared to (a) a cDMARD (methotrexate) and (b) a treatment pathway starting with adalimumab monotherapy via subcutaneous injection. Methotrexate was selected as the primary comparator to contrast sarilumab's performance against the historical 


\section{FIGURE 1 Model Framework}

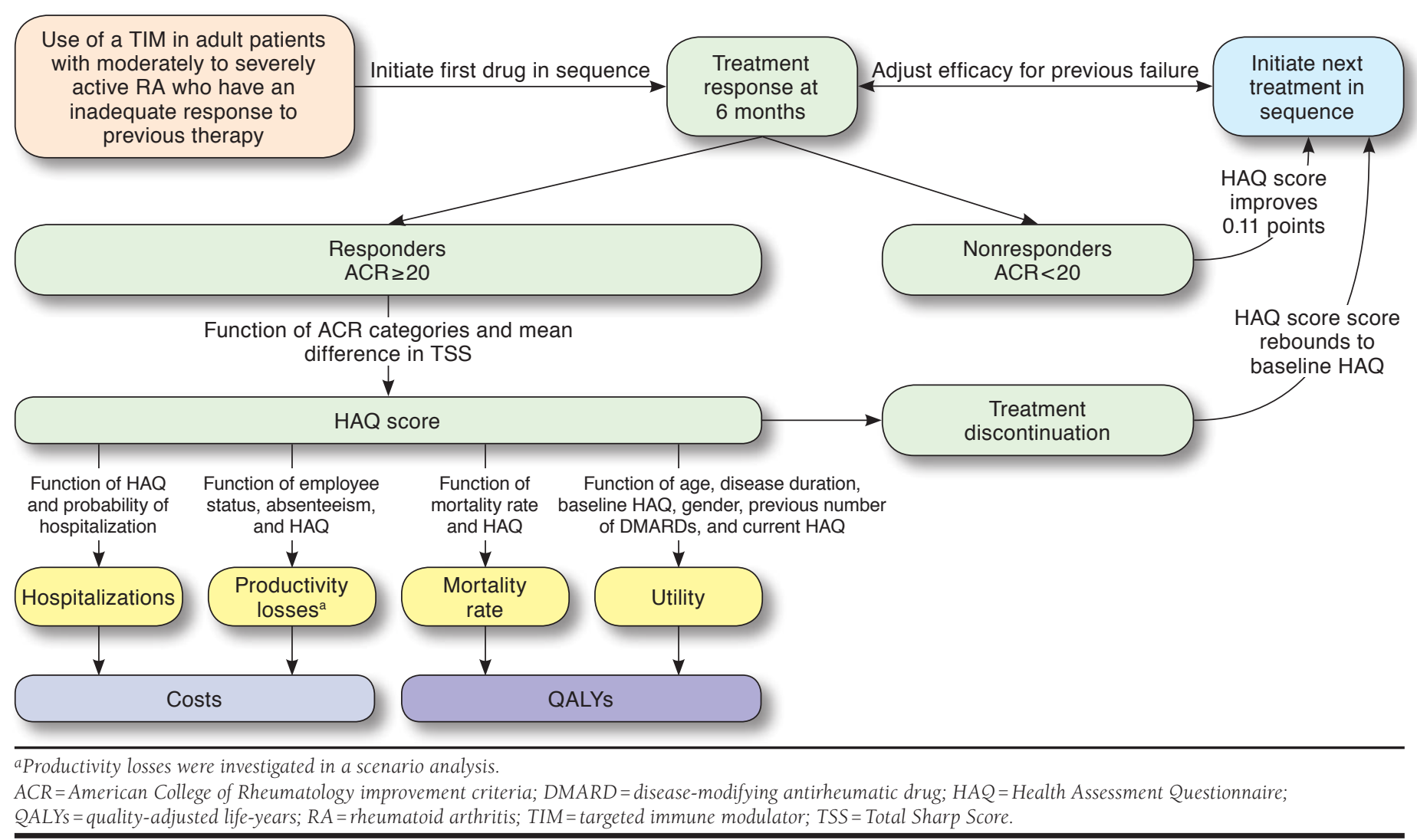

standard treatment. Adalimumab monotherapy was selected as a secondary comparator because it is the TIM leader by market share and has head-to-head evidence compared with sarilumab. ${ }^{7,12}$ The decision to focus on monotherapy was due to the head-to-head trial evidence available and superior outcomes observed. Further rationale on comparator selection can be found elsewhere. ${ }^{7}$

\section{Model Structure}

To promote model transparency, detailed documentation of the model can be found elsewhere. ${ }^{7}$ The model framework, depicted in Figure 1, was developed in Microsoft Excel (Microsoft, Redmond, WA). After starting a TIM, the ACR improvement criteria categories from the network metaanalysis were correlated to HAQ improvements. ${ }^{14,15}$ In addition to relating ACR response to the HAQ score, the model framework related the HAQ score to joint damage and radiographic progression, as measured through the modified TSS (mTSS). ${ }^{16}$ Thus, HAQ scores were simulated through separate contributions of ACR and mTSS, given baseline characteristics of the cohort. A lower HAQ score suggests lower RA disease activity and better overall functioning. ${ }^{17}$
Consistent with previous U.S. and European peer-reviewed RA models, ${ }^{14,16,18-20}$ the HAQ was the primary metric that was correlated with components of QALY (utility and life-years gained), as well as health care utilization and productivityrelated costs. A more favorable (lower) HAQ score is associated with a higher utility, lower likelihood of RA-related mortality, lower hospitalization rates, and higher productivity. The simulated utility score and mortality were used to calculate the QALYs gained. The simulated hospitalization rate factored into total costs. The simulated productivity losses were incorporated in the modified societal perspective. The relationship between HAQ and mortality, utility, health care utilization, and productivity can be found in Appendix B (available from the authors at http://www.ucdenver.edu/academics/colleges/ pharmacy/Departments/ClinicalPharmacy/DOCPFaculty/A-G/ Pages/CampbellJonathanDPhD.aspx).

Because it is common for patients to cycle through multiple therapies until they find the one they best respond to, the model allowed patients who discontinued a TIM to switch therapies up to 3 times. Patients could discontinue a therapy either due to lack of effectiveness (ACR score less than 20 in the 


\section{Assessing the Value of Sarilumab Monotherapy for Adults with Moderately \\ to Severely Active Rheumatoid Arthritis: A Cost-Effectiveness Analysis}

\section{FIGURE 2 Model Sequential Treatment Pattern}

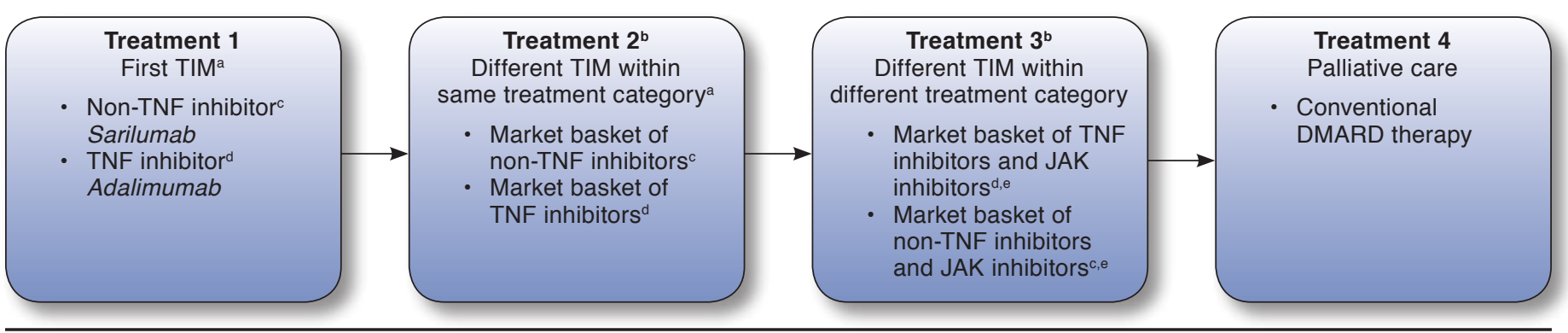

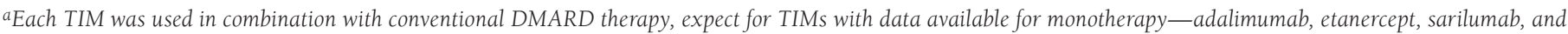
tocilizumab (intravenous).

bAll therapies in the market basket were averaged and were thus weighted equally.

'Non-TNF inhibitors include rituximab, abatacept (intravenous), abatacept (subcutaneous), tocilizumab (intravenous), tocilizumab (subcutaneous), and sarilumab.

dTNF inhibitors include adalimumab, certolizumab pegol, etanercept, golimumab (intravenous), golimumab (subcutaneous), and infliximab.

eJAK inhibitors include tofacitinib and baricitinib.

DMARD = disease-modifying antirheumatic drug; JAK=Janus kinase; TIM=targeted immune modulator; TNF= tumor necrosis factor .

first 6-month cycle) and/or the occurrence of adverse events. The sequential treatment pathway is outlined in Figure 2.

The model was validated following standard practices in the field. ${ }^{21}$ All mathematical functions were tested to ensure that they were consistent with the results. Exploratory analyses were conducted using null input values to ensure that the model was producing findings consistent with expectations. Further, 2 independent modelers tested the mathematical functions, inputs, and corresponding outputs. Model validation was conducted in terms of comparisons with other RA model findings examined in the Discussion section.

\section{Model Inputs}

Model inputs were estimated from a network meta-analysis described elsewhere, ${ }^{7}$ as well as from published literature. Cost inputs included drug costs (acquisition, administration, and monitoring); health care utilization costs; adverse event costs; and productivity costs. Drug acquisition costs were calculated using WAC from RED BOOK. ${ }^{8}$ These WAC prices were discounted using the most recent 4-quarter data average WAC and net price data from SSR Health that combined information on net U.S. dollar sales net of discounts, rebates, concessions to wholesalers and distributors, and patient assistance programs, with information on unit sales to derive net pricing at the unit level across all payers.

An average discount for each drug was calculated where available based on sales data in SSR Health. ${ }^{22}$ Sarilumab had not been on the market long enough to obtain a valid discounted cost estimate from SSR Health; therefore, the discount assumed for sarilumab was the average discount observed for drugs in the same drug class (20\% off). ${ }^{22}$ The undiscounted and discounted annual drug costs for the drugs of interest (sarilumab, adalimumab, and the cDMARD) can be found in Table 1 . Appendix $C$ includes the undiscounted and discounted annual drug costs for subsequent drugs in the treatment pathway (available from the authors at http://www.ucdenver.edu/academics/colleges/pharmacy/Departments/ClinicalPharmacy/ DOCPFaculty/A-G/Pages/CampbellJonathanDPhD.aspx). Costs associated with administration and monitoring were also included.

Drugs administered subcutaneously included the cost for an annual office visit for training on self-administration and for 1 subcutaneous administration. Drugs administered intravenously included the cost for an intravenous infusion administered in a physician's office. Drugs administered orally did not have costs associated with administration. Drug monitoring included office visits, tuberculosis tests, liver tests, and complete blood count tests. The package insert for each therapy was reviewed to determine which monitoring activities were appropriate for each therapy.

Health care utilization costs included costs associated with hospitalizations and office visits. Two severe adverse events (serious infections and tuberculosis infections) were assumed to affect costs. Productivity costs due to potential reduced absenteeism and employment were included in the modified societal perspective. ${ }^{23-25}$ The average hourly wage from the Bureau of Labor Statistics was used to monetize productivity gains/losses. ${ }^{26,27}$ Unit costs assigned to administration, monitoring, health care utilization, adverse events, and productivity can be found elsewhere. ${ }^{7}$

Clinical inputs included treatment response, radiographic progression, mortality, and utilities. ${ }^{7}$ Clinical inputs were retrieved from a network meta-analysis, ${ }^{7}$ as well as from published literature detailed in the Discussion section. Treatment 
TABLE 1 Base-Case Drug Costs and Clinical Signals for Drugs of Interest

Annual Drug Costs

\begin{tabular}{|c|c|c|c|c|c|}
\hline Drug & Administration & \multicolumn{2}{|c|}{ Undiscounted Annual Drug Cost, ${ }^{a} \$$} & \multicolumn{2}{|c|}{ Discounted Annual Drug Cost,$^{b} \$$} \\
\hline Sarilumab & SC & \multicolumn{2}{|c|}{39,000} & \multicolumn{2}{|c|}{31,200} \\
\hline Adalimumab & SC & \multicolumn{2}{|c|}{57,736} & \multicolumn{2}{|c|}{40,415} \\
\hline cDMARD (methotrexate) & Oral & \multicolumn{2}{|c|}{1,155} & \multicolumn{2}{|c|}{1,155 (generic) } \\
\hline \multicolumn{6}{|l|}{ Clinical Signals } \\
\hline Drug & $\begin{array}{c}\mathrm{ACR}<20 \\
\%\end{array}$ & $\begin{array}{c}\text { ACR } 20-50 \\
\%\end{array}$ & $\begin{array}{c}\text { ACR } 50-70 \\
\%\end{array}$ & $\begin{array}{c}\text { ACR } 70-100 \\
\%\end{array}$ & $\begin{array}{l}\text { TSS Median } \\
\text { Difference }\end{array}$ \\
\hline$\underline{\text { Sarilumab }}$ & 28 & 25 & 20 & 27 & -2.09 \\
\hline Adalimumab & 43 & 25 & 16 & 16 & -2.30 \\
\hline cDMARD (methotrexate) & 73 & 16 & 8 & 4 & 0.00 \\
\hline
\end{tabular}

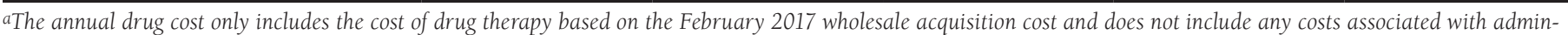

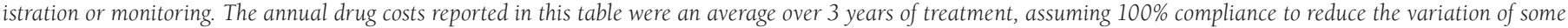
TIMs loading dosing schedule.

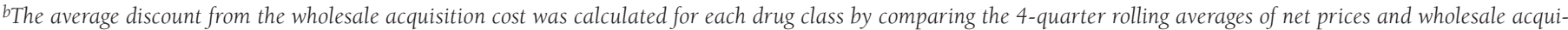
sition cost per unit.

$A C R=$ American College of Rheumatology improvement criteria; $c D M A R D=$ conventional disease-modifying antirheumatic drug; $S C=$ subcutaneous; TIM = targeted immune modulator; TSS = Total Sharp Score.

response was measured by ACR score and was related to HAQ scores using a previously published relationship..$^{14,15}$ The model also accommodated the association between joint damage and HAQ scores as measured through mTSS. ${ }^{16}$ HAQ scores were then linked to utility and mortality using previously published relationships. ${ }^{18,28}$ In addition, a disutility $(-0.156)$ was assigned to individuals who experienced a severe adverse event. ${ }^{29}$ The disutility lasted 1 month for those who experienced a serious infection and 2 months for those who contracted tuberculosis. ${ }^{29,30}$ Table 1 presents the ACR categories and mTSS mean difference for each drug of interest (sarilumab, adalimumab, and the cDMARD). Appendix D contains the ACR categories and mTSS mean difference for subsequent drugs in the treatment pathway (available from the authors at http://www.ucdenver.edu/academics/colleges/ pharmacy/Departments/ClinicalPharmacy/DOCPFaculty/A-G/ Pages/CampbelljonathanDPhD.aspx). A full description of all other clinical inputs can be found elsewhere. ${ }^{7}$

\section{Sensitivity Analyses}

To assess input variation and uncertainty, a probabilistic sensitivity analysis was conducted to vary parameter estimates across their plausible ranges simultaneously. Ranges were informed by $95 \%$ confidence intervals where available. When confidence intervals were not available, plausible ranges were used. One thousand paired estimates of incremental cost and incremental effect from the U.S. health sector payer perspective were calculated. Beta distributions were used for proportions, including treatment response. Normal distributions were assumed for measures of radiographic progression. Gamma distributions were assumed for rates, including discontinuation and adverse events and cost inputs.

\section{Scenario Analyses}

We conducted a threshold analysis to identify the annual sarilumab price that would meet value thresholds of $\$ 50,000$, $\$ 100,000$, and $\$ 150,000$ per QALY gained as compared with the cDMARD from a health sector payer perspective. We also ran the model using a 3-year time horizon instead of the lifetime time horizon used in the base case, since shorter-term results might interest some payers more than the lifetime time horizon assumed in the base case.

This study was determined to not be human subjects research by the Colorado Multiple Institutional Review Board.

\section{Results}

\section{Deterministic Results}

Over a lifetime time horizon, a treatment pathway starting with sarilumab resulted in 17.16 life-years and 13.66 QALYs (Table 2). This equates to more life-years and QALYs than a treatment pathway starting with the cDMARD or adalimumab therapy. Lifetime costs to the payer were higher for the sarilumab treatment pathway, which assumed a discount for sarilumab based on observed discounts for other drugs in its drug class, as compared with the cDMARD, but lower compared with the lifetime payer costs for adalimumab. These cost trends were consistent with the societal perspective analysis as well. When compared with the cDMARD, the sarilumab treatment pathway resulted in an incremental cost-effectiveness ratio of $\$ 227,308$ per QALY gained from the health sector payer perspective and $\$ 191,414$ per QALY gained from the restricted societal perspective. When compared with the adalimumab treatment pathway, the sarilumab treatment pathway was dominant (less costly and more effective) from both perspectives. 


\begin{tabular}{|c|c|c|c|c|c|}
\hline TABLE : & \multicolumn{5}{|c|}{$\begin{array}{l}\text { Deterministic Results over } \\
\text { Lifetime Time Horizon }\end{array}$} \\
\hline \multicolumn{6}{|c|}{ Model-Generated Outcomes } \\
\hline Treatment & $\begin{array}{l}\text { Cost: Health } \\
\text { Sector Payer } \\
\text { Perspective } \\
\$ \\
\end{array}$ & \multicolumn{2}{|c|}{$\begin{array}{c}\text { Cost: Societal } \\
\text { Perspective } \\
\$\end{array}$} & Life-Years & QALYs \\
\hline Sarilumab & 492,073 & \multicolumn{2}{|c|}{633,680} & 17.16 & 13.66 \\
\hline Adalimumab & 535,667 & \multicolumn{2}{|c|}{688,819} & 17.05 & 13.35 \\
\hline $\begin{array}{l}\text { CDMARD } \\
\text { (methotrexate) }\end{array}$ & 62,587 & \multicolumn{2}{|c|}{272,014} & 16.54 & 11.77 \\
\hline \multicolumn{6}{|c|}{ Incremental Results (Incremental Cost per QALY Gained) } \\
\hline Perspective & \multicolumn{2}{|c|}{ Treatment } & \multicolumn{2}{|c|}{$\begin{array}{l}\text { Comparator: } \\
\text { cDMARD, \$ }\end{array}$} & $\begin{array}{l}\text { Comparator: } \\
\text { Adalimumab }\end{array}$ \\
\hline \multirow{2}{*}{$\begin{array}{l}\text { Health sector } \\
\text { payer }\end{array}$} & \multicolumn{2}{|l|}{ Sarilumab } & \multicolumn{2}{|c|}{227,308} & Dominant \\
\hline & \multicolumn{2}{|c|}{ Adalimumab } & \multicolumn{2}{|c|}{298,877} & Reference \\
\hline \multirow[t]{2}{*}{ Societal } & \multicolumn{2}{|l|}{ Sarilumab } & \multicolumn{2}{|c|}{191,414} & Dominant \\
\hline & \multicolumn{2}{|c|}{ Adalimumab } & \multicolumn{2}{|c|}{263,325} & Reference \\
\hline
\end{tabular}

cDMARD = conventional disease-modifying antirheumatic drug; $Q A L Y=$ qualityadjusted life-years.

\section{Sensitivity Analyses Results}

Results from the probabilistic sensitivity analyses are displayed in Table 3 and Appendices E and F (available from the authors at http://www.ucdenver.edu/academics/colleges/ pharmacy/Departments/ClinicalPharmacy/DOCPFaculty/A-G/ Pages/CampbelljonathanDPhD.aspx). Even when accounting for input variation and uncertainty, none of the Monte Carlo iterations resulted in an incremental cost-effectiveness ratio less than $\$ 100,000$ per QALY gained when comparing sarilumab with the cDMARD. At a threshold of $\$ 250,000$ per QALY gained, the sarilumab treatment pathway was cost-effective $68 \%$ of the time compared with the cDMARD. Conversely, when sarilumab was compared with adalimumab, sarilumab was cost-effective $100 \%$ of the time, even at thresholds as low as $\$ 50,000$ per QALY gained.

\section{Scenario Analyses Results}

Results from the threshold analysis suggest that a $41 \%$ discount from the current WAC would be needed to reach a value threshold of $\$ 150,000$ per QALY gained. Discounts of $60 \%$ and $78 \%$ would be needed to reach value thresholds of $\$ 100,000$ and $\$ 50,000$ per QALY gained, respectively. Assuming a 3-year time horizon, the incremental cost-effectiveness ratio for sarilumab was approximately $\$ 366,000$ per QALY gained compared with the cDMARD from the health sector payer perspective. Sarilumab remained dominant over adalimumab with a 3-year time horizon.

\section{Discussion}

This analysis estimated the lifetime cost-effectiveness of a treatment strategy that started with sarilumab monotherapy for adult patients with moderately to severely active RA who had had an inadequate response to cDMARD therapy. The primary cost-effectiveness comparison was between a treatment strategy that started with sarilumab monotherapy versus cDMARD therapy; however, a comparison was also made between sarilumab monotherapy and the TIM market leader (adalimumab monotherapy). To our knowledge, this is the first economic evaluation to forecast the long-term costs and outcomes of sarilumab, which appears to be a highly effective and costly addition to the RA treatment armamentarium. We used rigorous and recommended RA cost-effectiveness analysis methods, but we also explicitly integrated the effects of treatment on radiographic progression and its consequent effect on utility and cost. To our knowledge, this is the only model that integrated these disease characteristics without access to patient-level data. ${ }^{17}$ Further, our model was developed through extensive engagement with manufacturers and other stakeholders, which we believe only heightens this contribution to the literature.

The deterministic results suggest that starting with a treatment strategy of sarilumab monotherapy provides substantial clinical benefit in comparison with the cDMARD alone. Additional costs of the sarilumab treatment strategy, however, translated into cost-effectiveness estimates that exceeded commonly cited thresholds of $\$ 50,000$ - $\$ 150,000$ per QALY gained when compared with cDMARDs. ${ }^{31}$ Compared with adalimumab, sarilumab was dominant (more effective and less costly).

The base-case results were generally robust to sensitivity analyses. When compared with the CDMARD, sarilumab was considered $1 \%$ likely to be cost-effective at a threshold of $\$ 150,000$ per QALY gained. Compared with adalimumab, sarilumab was $100 \%$ likely to be cost-effective at a threshold as low as $\$ 50,000$ per QALY gained.

Our study is the first study we are aware of that has produced cost-effectiveness estimates for sarilumab monotherapy since its approval. The recent RA model available online by Innovation and Value Initiative does not allow the user to evaluate monotherapy agents nor does it include sarilumab as monotherapy or in combination with cDMARDs. ${ }^{32} \mathrm{~A}$ previous study has used cost-effectiveness modeling to compare tocilizumab monotherapy with adalimumab monotherapy in biologic-naive patients. ${ }^{14}$ Our model is structurally similar and has similar population characteristics, yet the model from the earlier study assumed a constant discontinuation rate, whereas our model assumed a lower and drug-specific discontinuation rate. Also, our model uses the HAQ score as a function of both ACR category and mTSS. Further, due to differences in drug costs over time, the drug costs in our model were higher than the drug costs used in the previous model. A United Kingdomfocused microsimulation model compared adalimumab combination therapy with cDMARDs. ${ }^{16}$ This study yielded 6.83 QALYs for the adalimumab combination therapy and 3.79 QALYs for the cDMARD therapy. ${ }^{16}$ Our base-case analysis reflects similar QALY differences between these 2 therapies. 


\section{Assessing the Value of Sarilumab Monotherapy for Adults with Moderately to Severely Active Rheumatoid Arthritis: A Cost-Effectiveness Analysis}

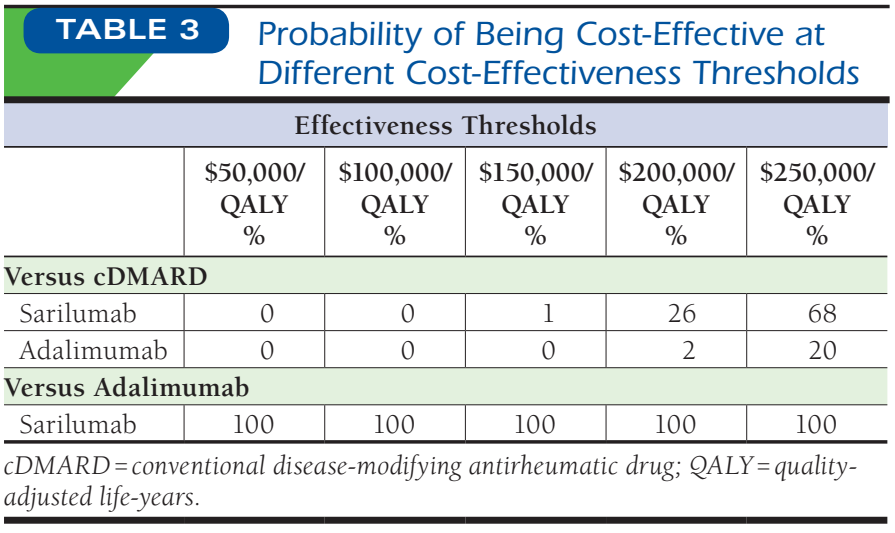

\section{Limitations}

This study has some limitations to consider. Sarilumab is new to the market, so data were premature to estimate the average discount from the WAC. In this analysis, we assumed the same discount as that observed for other drugs in the same class (20\%). Our results show that discount rates for sarilumab would need to be greater than the average discount observed for drugs in its same class to obtain incremental cost-effectiveness ratios below commonly cited thresholds of $\$ 50,000-\$ 150,000$ per QALY gained when compared with the cDMARD. Using the average discount observed for drugs in its same class, sarilumab was cost-effective compared with adalimumab.

In clinical practice, treatment choice is often based on patients' individual characteristics and risk factors, which may not be consistent with the sequential treatment pattern used in our model. However, exploratory analyses were conducted that assumed different treatment pathways, and the results and conclusions were robust. Also, TIM adherence was not included in this evaluation over and above that of TIM discontinuation and TIM switching, although simulated discontinuation rates were similar to those reported in observational studies.

Although tocilizumab is in the same drug class as sarilumab, this evaluation did not directly compare sarilumab with tocilizumab (administered either subcutaneously or intravenously) due to immature evidence (i.e., lack of head-to-head evidence). Future research should calculate the cost-effectiveness of sarilumab compared with tocilizumab once head-to-head data are available and the discount rate for sarilumab is known.

\section{Conclusions}

This analysis indicates that starting with a treatment strategy of sarilumab monotherapy results in better health outcomes than cDMARDs; however, the added cost, assuming class-level net pricing, led to cost-effectiveness estimates above commonly cited thresholds. Discounts required to achieve these thresholds are greater than the class-level applied discounts from the WAC assumed for sarilumab. Compared with adalimumab monotherapy, sarilumab monotherapy was more favorable and resulted in better health outcomes at a lower cost. This evaluation is important to inform stakeholders of the value of sarilumab compared with relevant alternative treatments to promote high value practices in health care.

\section{Authors}

MELANIE D. WHITTINGTON, PhD; R. BRETT MCQUEEN, PhD; and JONATHAN D. CAMPBELL, PhD, Department of Clinical Pharmacy, University of Colorado Anschutz Medical Campus, Aurora. DANIEL A. OLLENDORF, PhD; RICHARD H. CHAPMAN, PhD; VARUN M. KUMAR, MBBS, MPH, MSc; PATRICIA G. SYNNOTT, MS, MALD; and FOLUSO AGBOOLA, MBBS, MPH, Institute for Clinical and Economic Review, Boston, Massachusetts.

AUTHOR CORRESPONDENCE: Jonathan D. Campbell, PhD, 12850 E. Montview Blvd., V20-1203, Aurora, CO 80045.

Tel.: 303.724.2886; E-mail: Jon.Campbell@UCDenver.edu.

\section{DISCLOSURES}

Funding for this research was contributed by the Institute for Clinical and Economic Review (ICER). Ollendorf, Chapman, Kumar, Synnott, and Agboola are employees of ICER, an independent organization that evaluates the evidence on the value of health care interventions, which is funded by grants from the Laura and John Arnold Foundation, Blue Shield of California Foundation, and the California HealthCare Foundation. The organization's annual policy summit is supported by dues from Aetna, AHIP, Anthem, Blue Shield of California, CVS Caremark, Express Scripts, Harvard Pilgrim Health Care, Omeda Rx, United Healthcare, Kaiser Permanente, Premera Blue Cross, AstraZeneca, Genentech, GlaxoSmithKline, Johnson \& Johnson, Merck, National Pharmaceutical Council, Takeda, Pfizer, Novartis, Lilly, and Humana.

This work is an extension of an analysis presented at the New England Comparative Effectiveness Public Advisory Council on March 24, 2017, where the authors received public feedback on the analysis, results, and effect of a value assessment for targeted immune modulators. At the time of presentation, sarilumab was still an investigational product; therefore, a price was not known, so cost-effectiveness estimates were not generated. Since the presentation of that material, additional evidence for sarilumab has become available. The additional evidence has been incorporated into this analysis to present cost-effectiveness estimates for sarilumab.

\section{ACKNOWLEDGMENTS}

The authors thank Margaret Webb, Noah Mwandha, and Erin Lawler of ICER for their contributions to this analysis. The authors also acknowledge Samuel McGuffin of the University of Colorado for his editorial contributions to the manuscript.

\section{REFERENCES}

1. Helmick CG, Felson DT, Lawrence RC, et al. Estimates of the prevalence of arthritis and other rheumatic conditions in the United States. Part I. Arthritis Rheum. 2008;58(1):15-25.

2. Crane MM, Juneja M, Allen J, et al. Epidemiology and treatment of newonset and established rheumatoid arthritis in an insured U.S. population. Arthritis Care Res (Hoboken). 2015;67(12):1646-55.

3. Huizinga TW, Pincus T. In the clinic. Rheumatoid arthritis. Ann Intern Med. 2010;153(1):ITC1-1-ITC1-15. 


\section{Assessing the Value of Sarilumab Monotherapy for Adults with Moderately to Severely Active Rheumatoid Arthritis: A Cost-Effectiveness Analysis}

4. Birnbaum H, Pike C, Kaufman R, Marynchenko M, Kidolezi Y, Cifaldi M. Societal cost of rheumatoid arthritis patients in the U.S. Curr Med Res Opin. 2010;26(1):77-90

5. Arthritis Foundation. Rheumatoid arthritis treatment. August 9, 2017 Available at: http://www.arthritis.org/about-arthritis/types/rheumatoidarthritis/treatment.php. Accessed November 15, 2018.

6. Fransen J, van Riel PL. The Disease Activity Score and the EULAR response criteria. Clin Exp Rheumatol. 2005;23(5 Suppl 39):S93-99.

7. Institute for Clinical and Economic Review. Targeted immune modulators for rheumatoid arthritis: effectiveness \& value. Evidence report. 2017. Available at: https://icer-review.org/wp-content/uploads/2016/08/NE_CEPAC_ RA_Evidence_Report_FINAL_040717.pdf. Accessed November 15, 2018.

8. Truven Health Analytics, IBM Watson Health. Micromedex RED BOOK. Database. Retrieved February 27, 2017. Available at: http://truvenhealth. com/Products/Micromedex/Product-Suites/Clinical-Knowledge/RED-BOOK Accessed November 15, 2018.

9. Rubenfire A. Rheumatoid arthritis drug prices on the rise. Modern Healthcare. April 1, 2016. Available at: http://www.modernhealthcare.com/ article/20160401/NEWS/160409993. Accessed November 15, 2018.

10. KEVZARA (sarilumab) injection, for subcutaneous use. sanofi-aventis U.S. Revised May 2017. Available at: https://www.accessdata.fda.gov/drugsatfda_docs/label/2017/761037s000lbl.pdf. Accessed November 15, 2018.

11. Genovese MC, Fleischmann R, Kivitz AJ, et al. Sarilumab plus methotrexate in patients with active rheumatoid arthritis and inadequate response to methotrexate: results of a phase III study. Arthritis Rheumatol. 2015;67(6):1424-37.

12. Burmester GR, Lin Y, Patel R, et al. Efficacy and safety of sarilumab monotherapy versus adalimumab monotherapy for the treatment of patients with active rheumatoid arthritis (MONARCH): a randomised, double-blind, parallel-group phase III trial. Ann Rheum Dis. 2017;76(5):840-47.

13. Curtis JR, Jain A, Askling J, et al. A comparison of patient characteristics and outcomes in selected European and U.S. rheumatoid arthritis registries. Semin Arthritis Rheum. 2010;40(1):2-14.ell.

14. Carlson JJ, Ogale S, Dejonckheere F, Sullivan SD. Economic evaluation of tocilizumab monotherapy compared to adalimumab monotherapy in the treatment of severe active rheumatoid arthritis. Value Health. 2015;18(2):173-79.

15. Gabay C, Emery P, van Vollenhoven R, et al. Tocilizumab monotherapy versus adalimumab monotherapy for treatment of rheumatoid arthritis (ADACTA): a randomised, double-blind, controlled phase 4 trial. Lancet. 2013;381(9877):1541-50

16. Stephens S, Botteman MF, Cifaldi MA, van Hout BA. Modelling the cost-effectiveness of combination therapy for early, rapidly progressing rheumatoid arthritis by simulating the reversible and irreversible effects of the disease. BMJ Open. 2015;5(6):e006560.

17. Bruce B, Fries JF. The Stanford Health Assessment Questionnaire: dimensions and practical applications. Health Qual Life Outcomes. 2003;1:20.

18. Wailoo AJ, Bansback N, Brennan A, Michaud K, Nixon RM, Wolfe F. Biologic drugs for rheumatoid arthritis in the Medicare program: a costeffectiveness analysis. Arthritis Rheum. 2008;58(4):939-46.

19. National Institute for Health and Care Excellence. Tocilizumab for the treatment of rheumatoid arthritis. Technology appraisal guidance [TA247] February 22, 2012. Available at: https://www.nice.org.uk/guidance/ta247. Accessed November 15, 2018.

20. Athanasakis K, Tarantilis F, Tsalapati K, Konstantopoulou T, Vritzali E, Kyriopoulos J. Cost-utility analysis of tocilizumab monotherapy in first line versus standard of care for the treatment of rheumatoid arthritis in Greece. Rheumatol Int. 2015;35(9):1489-95.
21. Eddy DM, Hollingworth W, Caro JJ, Tsevat J, McDonald KM, Wong JB. Model transparency and validation: a report of the ISPOR-SMDM Modeling Good Research Practices Task Force-7. Med Decis Making. 2012;32(5):733-43.

22. SSRHealth. U.S. brand Rx net price. Access restricted document. 2016. Available at: http://www.ssrhealth.com/us-brand-rx-net-price-tool/. Accessed November 15, 2018.

23. Kavanaugh A, Smolen JS, Emery P, et al. Effect of certolizumab pegol with methotrexate on home and work place productivity and social activities in patients with active rheumatoid arthritis. Arthritis Rheum. 2009;61(11):1592-600.

24. Osterhaus JT, Purcaru O. Discriminant validity, responsiveness and reliability of the arthritis-specific Work Productivity Survey assessing workplace and household productivity within and outside the home in patients with axial spondyloarthritis, including nonradiographic axial spondyloarthritis and ankylosing spondylitis. Arthritis Res Ther. 2014;16(4):R164.

25. Han C, Li N, Peterson S. Minimal important difference in HAQ: a validation from health economic perspectives in patient with rheumatoid arthritis using real-world data. Paper presented at the 2015 American College of Rheumatology/Association of Rheumatology Health Professionals Annual Scientific Meeting; November 6-11, 2015; San Francisco, CA.

26. Bureau of Labor Statistics. May 2017 national occupational employment and wage estimates United States. Available at: http://www.bls.gov/oes/current/oes_nat.htm. Accessed November 15, 2018.

27. Bureau of Labor Statistics. Labor force statistics from the Current Population Survey. 2017. Available at: http://www.bls.gov/cps/cpsaat03.htm. Accessed November 15, 2018.

28. Wolfe F, Michaud K, Gefeller O, Choi HK. Predicting mortality in patients with rheumatoid arthritis. Arthritis Rheum. 2003;48(6):1530-42.

29. National Institute for Health and Care Excellence. Adalimumab, etanercept, inflfliximab, certolizumab pegol, golimumab, tocilizumab and abatacept for rheumatoid arthritis not previously treated with DMARDs or after conventional DMARDs only have failed. Technology appraisal guidance [TA375]. January 26, 2016. Available at: https://www.nice.org.uk/guidance/ta375. Accessed November 27, 2018

30. Centers for Disease Control and Prevention. Treatment for TB disease. April 5, 2016. Available at: http://www.cdc.gov/tb/topic/treatment/tbdisease. htm. Accessed November 15, 2018.

31. Neumann PJ, Cohen JT, Weinstein MC. Updating cost-effectivenessthe curious resilience of the \$50,000-per-QALY threshold. N Engl J Med. 2014;371(9):796-97.

32. Innovation and Value Initiative. IVI-RA Value Model. 2017. Available at: http://www.thevalueinitiative.org/ivi-ra-value-model/. Accessed November $15,2018$.

33. Frayer CD, Gu Q, Ogden CL. Anthropometric reference data for children and adults: United States, 2007-2010. National Center for Health Statistics. Vital Health Stat 11. 2012;(252):1-48. Available at: https://www.cdc.gov/nchs/ data/series/sr_11/srl1_252.pdf. Accessed November 15, 2018.

34. Lillegraven S, Prince FH, Shadick NA, et al. Remission and radiographic outcome in rheumatoid arthritis: application of the 2011 ACR/ EULAR remission criteria in an observational cohort. Ann Rheum Dis. 2012;71(5):681-86.

35. Breedveld FC, Weisman MH, Kavanaugh AF, et al. The PREMIER study: a multicenter, randomized, double-blind clinical trial of combination therapy with adalimumab plus methotrexate versus methotrexate alone or adalimumab alone in patients with early, aggressive rheumatoid arthritis who had not had previous methotrexate treatment. Arthritis Rheum. 2006;54(1):26-37.

36. Symmons DP, Silman AJ. The Norfolk Arthritis Register (NOAR). Clin Exp Rheumatol. 2003;21(5 Suppl 31):S94-99. 